\title{
AN AI\&ML BASED APPLICATION FOR VISUALLY IMPAIRED PEOPLE
}

\author{
Uma Yadav \\ Department of CSE \\ G. H. Raisoni \\ College of Engineering, \\ Nagpur, India
}

\author{
Shweta Bondre \\ Department of CSE \\ G. H. Raisoni \\ College of Engineering \\ Nagpur, India
}

\author{
Priya Maidamwar \\ Department of CSE \\ G. H. Raisoni \\ College of Engineering \\ Nagpur, India
}

\author{
Prashant Khobragade \\ Department of CSE \\ G. H. Raisoni \\ College of Engineering \\ Nagpur, India
}

\begin{abstract}
For achieving dreams, goals and objective in life, independence plays a very important role. Visually impaired people realize themselves laborious to travel out severally. There square measure thousands of visually impaired individuals during this world $U N$ agency square measure sometimes in would like of serving to palms. For decades, there square measure varied devices used, the white cane has become a widely known attribute to blind people's navigation and later efforts are created to reinforce the cane by adding a unique device. Blind individuals face downside once they walk on the road or stairs employing a white cane, however they need sharp somatosense sensitivity. The digital walking stick can assist the blind individuals by supporting additional handy manner of existence. There square measure various steering structures for visually impaired travelers to navigate quickly and completely against boundaries and completely different dangers two-faced. Commonly, a blind user contains a white cane or a guidance canine as their quality resource. With the advances of recent technology several distinctive styles of devices square measure support quality of blind, called Electronic Travel Aids (ETAs). The essential feature of ETA for the visually handicapped person is to urge statistics at the shape of the road and therefore the placement of limitations once they square measure in unknown locations. With these facts, they have to reach their locations, avoiding sudden boundaries. The principle intention of this is to create, contribute our information and offerings to the individuals of blind and disable society.
\end{abstract}

Keywords - GPS, Blind, Navigation, Impaired Vision

\section{INTRODUCTION}

Machine learning is associate application of AI that has systems the power to mechanically learn and improve from expertise while not being expressly programmed. Machine learning may be a class and scientific study of sorts of algorithmic program that permits software package applications to become a lot of correct in predicting outcomes of any utility program or the important life downside. The basic vital factor of machine learning is to make algorithms which can receive knowledge input file computer file from close associated use applied mathematics analysis to predict an output by giving the solutions whereas change outputs as new data becomes on the market. There square measure over 284 million folks that square measure visually impaired and there square measure over 39 million folks that square measure all blind. The lack of visual capabilities has restricted these people from utterly perceiving their immediate surroundings that has potential safety considerations and conjointly lowers their quality of life since they need to believe some form of aid to urge around.

Every time for his or her day to day wants they need to be addicted to others that troubles them loads. So counting on this issue we've got created associate voice primarily based application victimisation OCR(Optical Character Recognition) which might minimize their dependency on others and facilitate them to travel where they require with none reasonably facilitate and even allow them to understand whether or not the placement they're looking for is correct or not. Optical Character Recognition (OCR) systems are effectively developed for the popularity of written script. The accuracy of OCR system in the main depends on the text preprocessing and segmentation algorithmic program getting used once the document is scanned it are often placed in any discretionary angle which might seem on the pc monitor at a similar angle[4], [10]. The construct of OCR would lead to providing the facilities the blind folks need. it'll make sure that the folks goes to the correct location by scanning the text and speech primarily based recognition system, the OCR can first off scan the text and it might convert the text into the speech 


\section{International Journal of Engineering Applied Sciences and Technology, 2019 \\ Vol. 4, Issue 7, ISSN No. 2455-2143, Pages 229-232 \\ Published Online November 2019 in IJEAST (http://www.ijeast.com)}

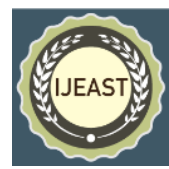

and a voice primarily based speech would be detected that may aware the person concerning the placement and he would return to grasp whether or not the placement he's looking for is reached or not. The person would be able to realize his current location by GPS(Global Position System) which might be conjointly helpful for him. thus during this approach atiny low mobile primarily based application by victimisation GPS and OCR would solve his dependency downside and would build him satisfy his daily wants.

\section{LITERATURE REVIEW}

Ivanov R. (2012) emphasized that Outdoor navigation of visually impaired people is based on data from the GPS maps and Geographic Information Systems (GIS) [1]. Such systems cannot be used for navigation in the regions where there are not any GPS maps or the maps are not sufficiently precise and detailed. In this article he has proposed an algorithm for realtime GPS track simplification. The test results show that the proposed algorithm (RSTS) has efficiency similar to the Douglas Peucker algorithm, which is regarded as the best for track simplification. The reduction of the number of points in different transportation modes, while keeping the shape of the route, is over $90 \%$. The size of the file describing the track is reduced more than 30 times. The algorithm finds the critical points of the route. This allows for navigation along the track so conversion of track to route is not needed. The algorithm is part of a low cost and widely accessible Java 2 Mobile Edition (J2ME) application for navigation of visually impaired. The drawback in the proposed article was that there was no voice based system which will guide the visually impaired person.

Koley et al. (2014) has used white canes to assist in obstacle detection \& dodging [11]. Here the goal of author was to make a transportable, straightforward less expensive system that may enable Blind peoples to travel through acquainted and unknown environments while not the help of guides. Many guidance device has been developed for vision impaired individuals, however these systems tends to be valuable, additionally create use of a shopper server approach. This Navigation system consists of 2 distinct components: sensing of the immediate setting for blind individuals to travel (e.g., obstacles and hazards) and navigating to remote destinations on the far side the straight off perceptible setting. The paper delineate here centered on the event and analysis of a Navigation system that produces use of GPS (the world Positioning System), voice and inaudible detector for obstacle detection. This system is Innovative however is extremely valuable and traditional individuals cannot afford shopping for this.

Moulton et al. (2009) has investigated the quality of a user centred shopper server approach for the event of a talking GPS system meant to fill a distinct segment for out of doors way finding [3]. The work resulted in a very operating model proof -of-concept system that uses a speech-recognition speechsynthesis interface. The model answer includes a custom internet application that accesses the Google maps API. The system is meant to be ascendable and protrusile with extra options like sensors for obstacle turning away and access to internet -based info like weather, train or bus timetable info. In order to avoid obstacles sensors are used that a hardware device are needed, there's an occasion of incidence of little accidents for the blind folks as they might not be ready to handle the hardware device.

Borenstein (1997) has introduced the GuideCane [2] , a completely unique device designed to assist blind or visually impaired travellers to navigate safely and quickly among obstacles and alternative hazards long-faced by blind pedestrians. The GuideCane, presently beneath development at the University of Michigan's Mobile artificial intelligence research laboratory, contains of a protracted handle and a detector head" unit that's hooked up at the distal finish of the handle. The detector head is mounted on a manageable however unpowered simple machine steering shaft throughout operation, the user pushes the sunshine weight Guide Cane previous him/herself unhearable detectors mounted on the sensor head discover obstacles and steer the device around it. The user feels the steering command as a really noticeable physical force through the handle and is ready to follow the guide Cane's path simply and with none aware effort. The drawback long-faced within the higher than mentioned plan is that the guide cane utilized in the project isn't an inexpensive choice for each common visually impaired person. And there's additionally a small risk of facing technical errors which may be fatal for the protection of a blind man.

Ertan et al. (1998) described a wearable navigation system supported a tactile directional show embedded within the back of Avast[5]. The system consists of a 4-by-4 array of micro motors for delivering tactile steering signals to the user's back, associate degree infrared primarily based input system for locating the user in associate degree setting, and a wearable laptop for route designing. User testing was conducted to guage the effectiveness of this method as a navigation guide for hawk-eyed users in associate degree unacquainted with research laboratory space. it's hoped that such a system is a helpful navigation guide for people with severe visual impairments in associate degree unacquainted with setting. Future work can address the precise problems regarding blind navigation. During getting dark or if there aren't any lights in an exceedingly area for ex. Theatre the projected system are going to be of no use in the slightest degree because the visually impaired person wouldn't be ready to find something or anyone within the close setting.

Tripathy et al.(2012) described that visually impaired folks had solely a restricted means that of accessing written material [9]. The accessibility of the large data bank that's obtainable 


\section{International Journal of Engineering Applied Sciences and Technology, 2019 \\ Vol. 4, Issue 7, ISSN No. 2455-2143, Pages 229-232 \\ Published Online November 2019 in IJEAST (http://www.ijeast.com)}

within the variety of written text is therefore terribly restricted for them. A visually impaired has 2 ancient ways that of accessing text information, they're the Braille script and audio tapes. However, even the help of those media isn't enough and therefore the blind need to think about the keen-sighted folks for mundane reading. This reliance and therefore the higher prices of manufacturing different format materials essentially scale back the fabric convenience during this work it's been projected to eradicate the on top of mentioned hindrance. Common problems with the present readers and user reviews are studied. Solutions to beat the problems of the present devices are thought over and a tool known as VIMPY- A Yapper for the Visually Impaired has been developed; it's a reader for the blind that helps reading any written A4 size physical document. it's a standalone device that uses its own software system, Vimpy OS an effort has been created to form a machine that converts physical documents containing written text to speech and provides different facilities like choices to avoid wasting text for later use, customise user expertise by sterilization pitch, volume, speed and additionally scan text files within the Devnagiri script etc.

Shinde et al. (2012) suggested that Optical Character Recognition (OCR) systems are effectively developed for the popularity of written script [8]. The accuracy of OCR system primarily depends on the text preprocessing and segmentation rule being employed. once the document is scanned it are often placed in any whimsical angle which might seem on the pc monitor at identical angle. This paper addresses the rule for correction of skew angle generated in scanning of the text document and a unique profile primarily based technique for segmentation of written text that separates the text in document image into lines, words and characters. So finally bearing on the higher than papers we tend to took the thought of exploitation Optical character recognition Associate in Nursingd exploitation the world positioning System(GPS) to produce an application for the visually impaired individuals because it is totally code primarily based it's simple and liberal to install and user friendly.

\section{PROPOSED SYSTEM}

System style focuses on the elaborate implementation of the possible system. It stress on translating style specifications to performance specification. System style has 2 phases of development logical and physical style. Throughout logical style part the analyst describes inputs (sources), out puts (destinations), databases (data sores) and procedures (data flows) beat a format that meets the uses necessities [4], [6]. The analyst additionally specifies the user wants and at grade that nearly determines the knowledge flow into and out of the system and therefore the data resources. Here the logical style is completed through dataflow diagrams and information style. The physical style is followed by physical style or writing [7]. Physical style produces the operating system by shaping the look specifications that tell the programmers specifically what the candidate system should do.

The programmers write the mandatory programs that settle for input from the user, perform necessary process on accepted knowledge through decision and turn out the desired report on a tough copy or show it on the screen[12]. Additionally the newest GPS technology is being employed. Upon the identification of categories and their responsibilities, user situations were then known and accustomed establishes collaborations and dependencies of those categories. These situations were additionally accustomed develop sequence diagrams. Finally the known categories were valid against the system design and system necessities. As a results of this method, it absolutely was determined that the project would arrange to develop a useful image victimization GPS and voice recognition technology.
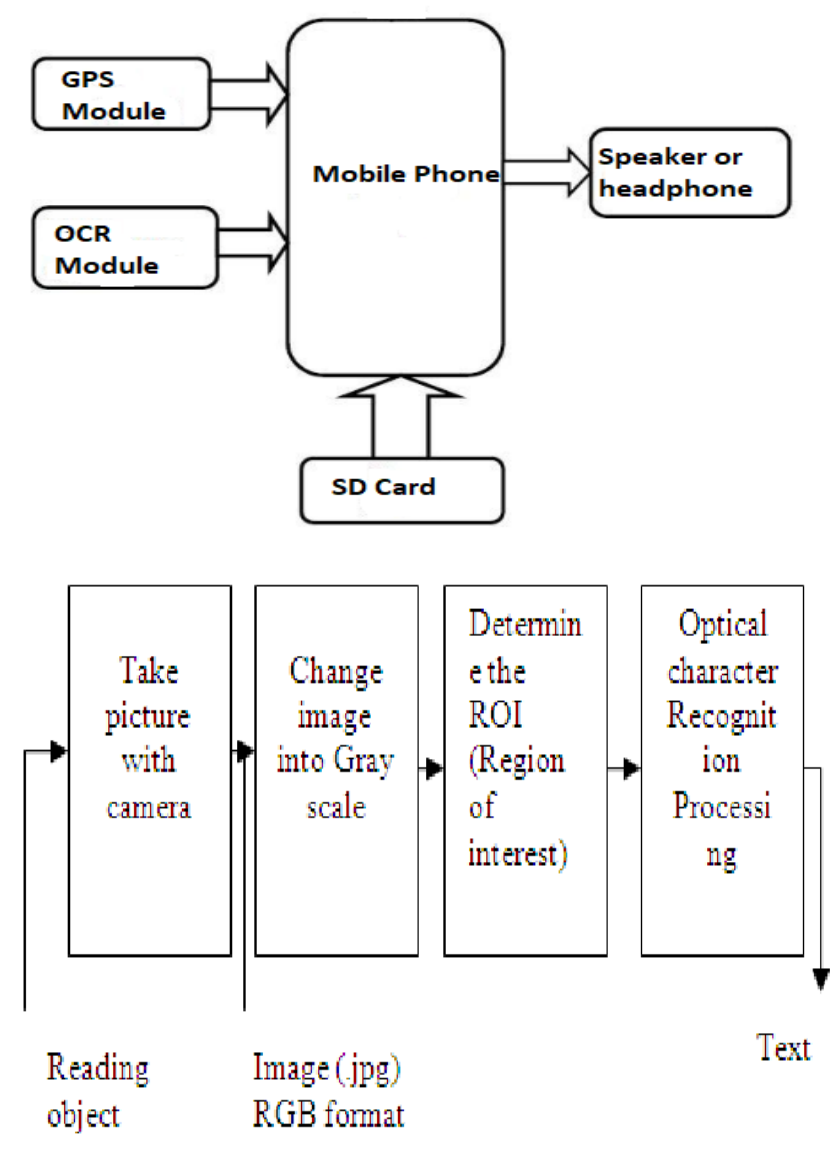

Fig 1: Sysem Diagram

\section{CONCLUSION}

The application made for visually impaired people can be very useful when they need to read an office poster or a 


\section{International Journal of Engineering Applied Sciences and Technology, 2019 \\ Vol. 4, Issue 7, ISSN No. 2455-2143, Pages 229-232 \\ Published Online November 2019 in IJEAST (http://www.ijeast.com)}

magazine or even a banknote. The use of application by blind users can be difficult but it doesn't create problem for people with low sight. Interaction between people with visual impairments and application was assured with help of touch commands on the screen and audio communication of application state, error and OCR results from smartphone to user. Voice recognition can also be used to give commands to application but in noisy environments it can give errors of understanding. Audio information is delivered at headphones because using the speaker can also create problems of understanding in noisy environments and can disturb other people. OCR function can be made locally, on the smartphone, but tests shows that it takes long time, so preferred method was the use of a remote webserver on the internet.

\section{REFERENCE}

[1] Ivanov R. (2012) Real-time GPS track simplification algorithm for outdoor navigation of Visually impaired\|, Journal of Network and Computer Applications, vol. 35, no. 5 , pp. $1559-1567$

[2] Borenstein, J. (1997) A Computerised Travel Aide for the Active Guidance of Blind Pedestrians, Proceedings of the IEEE International Conference on Robotics and Automation, Albuquerque, NM, Apr. 21-27, pp. 12831288.

[3] Moulton B., Pradhan G., Chaczko Z., (2009)"Voice Operated Guidance Systems for Vision Impaired People: Investigating a User - Centered Open Source Model", International Journal of Digital Content Technology and its Applications, Volume 3 Number 4, December :pp 60 68.

[4] Long, S., Aust, D., Abowd, G.D., Atkeson, C. (1996). Cyberguide: prototyping context-aware mobile applications. In CHI '96 conference companion, pp 293294

[5] Ertan, S., Lee, C., Willets, A., Tan, H., Pentland, A. (1998). A wearable haptic navigation guidance system. 2nd international symposium on wearable computer,Pittsburgh, PA, pp $164-165$.

[6] Golding, A.R., Lesh, N. (1999). Indoor navigation using a diverse set of cheap, wearable sensors. In: third international symposium on wearable computers. San Francisco, CA, pp 29-36.

[7] shttps://www.researchgate.net/publication/286800382_ OCR_Application_on_Smartphone_for_Visually_Impaire d People.

[8] Shinde A., Chougule D.G., Text Pre-processing and Text Segmentation for OCR. IEEE International Conference, January 2012.

[9] Tripathy A., Pathak A. , Rodrigues A. , Chaudhari C., Computer Engineering, Don Bosco Institute of
Technology, Mumbai, India, VIMPY - A Yapper for the visually impaired, Published in: 2012 World Congress on Information and Communication Technologies .

[10] Sreenivasan D., Poonguzhali S., (2013) An Electronic Aid for Visually Impaired in Reading Printed Text, International Journal of Scientific \& Engineering Research, Volume 4, Issue 5, ISSN 2229-5518, pp. 198203.

[11] Koley S., Mishra R., (2014)— Voice Operated Outdoor Navigation System for Visually Impaired Persons, International Journal of Engineering Trends and Technology- Vol.3.

[12] Anagnostopoulos C., Anagnostopoulos I., Loumos V., \& Kayafas E. 2006. A License Plate Recognition Algorithm for Intelligent Transportation System Applications.., IEEE Transactions on Intelligent Transportation Systems, pp. 377- 399 . 